\title{
Dynamic Model of 6 DOF for a Catamaran
}

\section{Homero J. Oria-Aguilera ${ }^{1}$, Yunier Valeriano-Medina ${ }^{2}$, Luis Hernández}

\author{
${ }^{1,2,3}$ Universidad Central "Marta Abreu" de Las Villas (UCLV) - Santa Clara, Villa Clara.
}

Email: homerooria@gmail.com, yunierv@uclv.edu.cu, luishs@uclv.edu.cu

\author{
Received: November $10^{\text {th }}, 2017$. \\ Accepted: January $06^{\text {th }}, 2018$. \\ Published: March $30^{\text {th }}, 2018$. \\ Copyright @2016 by authors and \\ Institute of Technology Galileo of \\ Amazon (ITEGAM). \\ This work is licensed under the \\ Creative Commons Attribution \\ International \\ License (CC BY 4.0). \\ http://creativecommons.org/licenses/by/4.0/

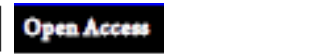

\begin{abstract}
The need to expand the exploitation and preservation of marine resources makes the interest in marine surface vehicles increases. Subjects such as modeling, simulation and control are now open research lines. In this research a nonlinear dynamic model of six degrees of freedom, which represents the main dynamic characteristics of two hull catamaran. The effect of environmental factors is included. The model has been obtained for the purpose of developing a training simulator for this vessel. In this paper the equations representing the dynamics of the vehicle from which the nonlinear model is obtained by applying an analytical and semi empirical method are defined. The validity of the model is found by simulation using MATLAB Simulink.
\end{abstract}

Keywords: Catamaran; Simulator; Dynamic model; Simulation.

\begin{abstract}
RESUMEN
La necesidad de expandir la explotación y preservación de los recursos marinos aumenta el interés en los vehículos de superficie marina. Temas tales como modelado, simulación y control ahora son líneas de investigación abiertas. En esta investigación se trata de un modelo dinámico no lineal de seis grados de libertad, que representa las características dinámicas principales de dos catamaranes de casco. El efecto de los factores ambientales está incluido. El modelo se ha obtenido con el propósito de desarrollar un simulador de entrenamiento para este buque. En este trabajo se definen las ecuaciones que representan la dinámica del vehículo a partir del cual se obtiene el modelo no lineal aplicando un método analítico y semi empírico. La validez del modelo se encuentra mediante simulación usando MATLAB Simulink.
\end{abstract}

Palabras Claves: Catamarán; Simulador; Modelo dinámico; Simulación.

\section{INTRODUCCIÓN}

El término vehículo de superficie engloba a barcos, embarcaciones de alta velocidad, así como otros vehículos que posean una estructura vacía que flote sobre la superficie con propósitos de transporte y navegación [1]. Específicamente, los catamaranes son embarcaciones de dos cascos, habitables o no, unidos entre sí por una estructura o plataforma [2].

El Centro de Investigación y Desarrollo de Simuladores (SIMPRO) y el Grupo de Automatización, Robótica y Percepción $(G A R P)$ se han propuesto desarrollar un simulador de entrenamiento para un vehículo de superficie tipo catamarán.

La aplicación debe asegurar un adecuado entrenamiento del personal en el manejo del vehículo para distintos tipos de maniobras, donde las condiciones del viento, de las corrientes marinas y del oleaje pueden ser alteradas. Para poder llevar a cabo este proyecto resulta necesario modelar la dinámica de la embarcación, así como de los factores medioambientales que lo afectan para evaluar su desempeño en los seis grados de libertad $(6 G D L)$. En esta tarea se ha aprovechado la experiencia de GARP de haber obtenido un modelo no lineal de $6 G D L$ para el vehículo autónomo subacuático $H R C$-AUV [3][4].

Los modelos se utilizan para la predicción y simulación en tiempo real, y para el diseño de observadores y controladores.

Atendiendo a su complejidad y al número de ecuaciones diferenciales que utilizan pueden distinguirse tres tipos de modelos [1]: modelo de simulación, modelo para el diseño del control y modelo para el diseño del observador. En la literatura se reportan numerosos ejemplos de investigaciones que se realizan con el propósito de obtener modelos dinámicos de 
vehículos marinos útiles en la simulación [5][6], así como en el diseño de los controladores y observadores [4][7-9].

El modelo dinámico de $6 G D L$ incluye el efecto de las perturbaciones marinas y el viento, así como el modelo de los actuadores que controlan la dirección del vehículo. En esta investigación se obtiene un modelo utilizando un procedimiento analítico y semi empírico, donde a partir de las características geométricas y estructurales de la embarcación se calculan algunos de los coeficientes hidrodinámicos. Otros fueron obtenidos a partir de experimentos [10] y tablas [11] referidas a vehículos que se consideran prototipos en las investigaciones científicas. La validez del modelo se evalúa mediante la simulación de un conjunto de maniobras típicas de vehículos marinos.

Este artículo sigue la siguiente estructura. En la Sección II se presenta la descripción general del vehículo. La estructura del modelo dinámico de $6 G D L$ se expone en la Sección III.

La Sección IV describe la obtención de los parámetros necesarios, así como el modelo de los actuadores. En la Sección $\mathrm{V}$, se presenta el cálculo de estos parámetros. El comportamiento mediante simulación del modelo ante distintas maniobras es mostrado en la Sección VI. Finalmente, las conclusiones son presentadas en la Sección VII.

\section{DESCRIPCIÓN GENERAL DEL CATAMARÁN DE SIMPRO}

La estructura del vehículo de superficie tipo catamarán, para el cual la empresa SIMPRO tiene previsto desarrollar un simulador, es similar a la del catamarán DELFIM [2].

Está compuesto por 2 flotadores o cascos, paralelos entre sí, unidos por estructuras delgadas ubicadas en popa y proa. Estos elementos sujetan, de igual manera, a un cuerpo cilíndrico que puede ser liberado cuando se desee. Estos cascos poseen forma cilíndrica y terminaciones oblicuas en proa.

El sistema de actuadores del catamarán está compuesto por dos hélices propulsadas por motores Yamaha E40XMH de 40 $h p$ cada uno. Estos motores incluyen una pequeña superficie vertical que origina un ángulo de deflexión en los mismos.

Los motores tienen dos modos de trabajo:

- Modo común, cuando las velocidades de giro de los motores son iguales.

- Modo diferencial, cuando las velocidades de giro de los motores son diferentes.

Los modos de trabajo de los motores permiten manipular la velocidad y orientación del vehículo. Si el catamarán sigue una línea recta, es debido a que los motores se encuentran trabajando en modo común y no existe ángulo de deflexión alguno. Si por el contrario, las maniobras presentan giros, es porque los motores se encuentran operando en modo diferencial y/o existe un valor de ángulo de deflexión.

Las especificaciones geométricas e inerciales son mostradas en la Tabla 2.

Como cada uno de los componentes de la estructura del catamarán generan fuerzas y momentos independientes, es necesario conocer la ubicación de estos con respecto al centro de gravedad del vehículo. Estos datos se reflejan en la Tabla 2.
Tabla 1: Características del catamarán de Simpro.

\begin{tabular}{ccc}
\hline Parámetros & Descripción & Valor \\
\hline $\mathrm{m}$ & Masa & $2300 \mathrm{~kg}$ \\
\hline$u_{0}$ & Velocidad crucero & $4.5 \mathrm{~m} / \mathrm{s}$ \\
\hline $\mathrm{n}$ & Revoluciones de los motores & $91 \mathrm{rps}$ \\
\hline $\mathrm{L}$ & Largo & $10 \mathrm{~m}$ \\
\hline $\mathrm{B}$ & Ancho & $2.82 \mathrm{~m}$ \\
\hline$D_{c}$ & Diametro de un flotador & $0.94 \mathrm{~m}$ \\
\hline $\mathrm{T}$ & Calado & $0.7 \mathrm{~m}$ \\
\hline $\mathrm{D}$ & Diametro de las helices & $0.23 \mathrm{~m}$ \\
\hline Parámetros & Descripción & Valor \\
\hline \hline$\delta r$ & Angulo de deflexion de los & $\pm 45^{\circ}$ \\
\hline \multicolumn{3}{c}{ motores } \\
\hline$I_{x x}$ & Momento de inercia & $2820 \mathrm{kgm}^{2}$ \\
\hline$I_{y y}$ & Momento de inercia & $1450 \mathrm{kgm}^{2}$ \\
\hline$I_{z z}$ & Momento de inercia & $1040 \mathrm{kgm}^{2}$ \\
\hline$I_{x z}$ & Momento de inercia & $680 \mathrm{kgm}^{2}$ \\
\hline
\end{tabular}

Fuente: [11].

Tabla 2: Distancias de cada elemento con respecto a la referencia del Catamarán.

\begin{tabular}{ccccc}
\hline & Casco 1 & Casco 2 & Motor 1 & Motor 2 \\
\hline $\mathrm{x}$ & 0 & 0 & $-3.88 \mathrm{~m}$ & $-3.88 \mathrm{~m}$ \\
\hline $\mathrm{y}$ & $-0.94 \mathrm{~m}$ & $0.94 \mathrm{~m}$ & $-0.94 \mathrm{~m}$ & $0.94 \mathrm{~m}$ \\
\hline $\mathrm{z}$ & 0 & 0 & 0 & 0 \\
\hline
\end{tabular}

Fuente: [11].

\section{MODELO DINÁMICO DE 6 GRADOS DE LIBERTAD PARA EL CATAMARÁN DE SIMPRO}

Para obtener el modelo dinámico no lineal de 6 grados de libertad de este vehículo, se utiliza un procedimiento analítico y semi empírico. Este método se basa en principios físicos junto con valores numéricos determinados mediante experimentos o calculo numérico.

Para describir la trayectoria de un vehículo marino de superficie es conveniente introducir dos sistemas de referencia: uno inercial con respecto a tierra $\{U\}$ y otro respecto al propio vehículo $\{C\}$ [2]. Las posiciones relativas de $\{U\}$ respecto a tierra o de $\{C\}$ respecto al catamarán pueden ser arbitrarias.

En la Figura 1, se muestra los sistemas de referencia $\{C\}$ y $\{U\}$ utilizados para el cálculo de las ecuaciones dinámicas del modelo. El origen del sistema de referencia $\{C\}$ coincide con el centro de gravedad. Por lo que la distancia entre el origen de coordenadas y el centro de flotabilidad del vehículo queda definido por el vector $\boldsymbol{r}_{\boldsymbol{B}}$.

La Tabla 3 resume la nomenclatura estándar empleada para describir el movimiento de vehículos marinos [12].

Tabla 3: Notación utilizada para vehículos marinos.

\begin{tabular}{cccc}
\hline Movimiento & Fuerza & Velocidad & Posición \\
\hline Avance & $X$ & $v$ & $x$ \\
\hline Desplazamiento lateral & $\gamma$ & $v$ & $y$ \\
\hline Arfada & $Z$ & $\omega$ & $z$ \\
\hline Balanceo & $K$ & $\vartheta$ & $\theta$ \\
\hline Cabeceo & $H$ & $q$ & $\psi$ \\
\hline Guiñada & $N$ & $r$ & \\
\hline
\end{tabular}

Fuente: Los Autores, (2015). 


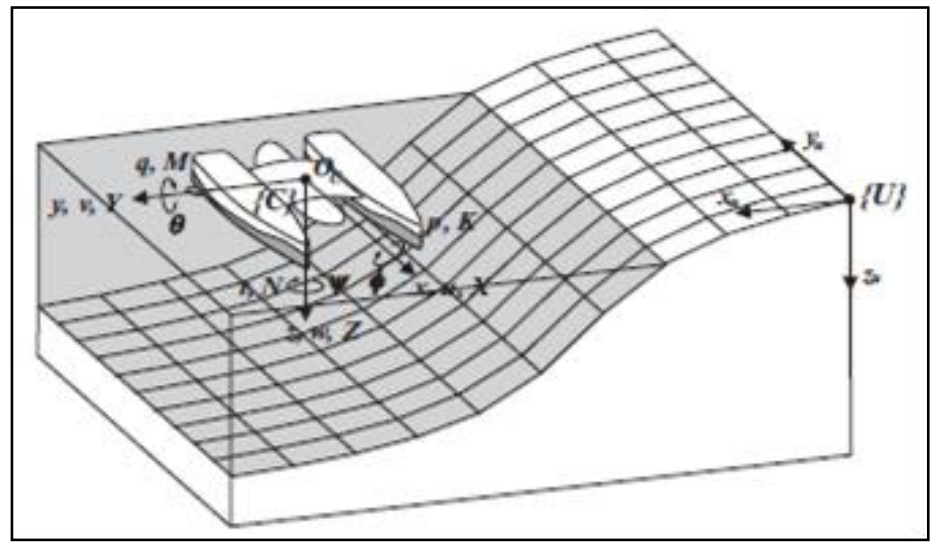

Figura 1: Sistemas de referencias $\{U\}$ y $\{C\}$.

Fuente: Los Autores, (2015).

Los seis grados de libertad del catamarán pueden ser divididos en dos grupos: el plano horizontal y el plano vertical. En la Tabla 4 aparecen los estados asociados a ambos planos.

Tabla 4: Notación utilizada en cada plano de los 6 grados de libertad.

\begin{tabular}{cc}
\hline Plano horizontal & Plano vertical \\
\hline $\boldsymbol{\eta}_{\mathbf{1}}=[x, y, \psi]^{T}$ & $\boldsymbol{\eta}_{\mathbf{2}}=[z, \phi, \theta]^{T}$ \\
\hline $\boldsymbol{\nu}_{\mathbf{1}}=[u, v, r]^{T}$ & $\boldsymbol{\nu}_{\mathbf{2}}=[\omega, p, q]^{T}$ \\
\hline $\boldsymbol{\tau}_{\mathbf{1}}=[X, Y, N]^{T}$ & $\boldsymbol{\tau}_{\mathbf{2}}=[Z, K, M]^{T}$ \\
\hline
\end{tabular}

Fuente: Los Autores, (2015).

Todos los estados dinámicos del catamarán se encuentran referenciados respecto al sistema $\{C\}$, con excepción de la posición, que se diferencia de los demás estados por estar referenciado respecto al sistema $\{U\}$.

La velocidad $\eta=\left[\begin{array}{ll}\eta_{1}, & \eta_{2}\end{array}\right]^{T}$ y la posición $\nu=\left[\nu_{1}\right.$, $\left.\nu_{2}\right]^{T}$ expresados en uno $\mathrm{u}$ otro sistema, pueden trasladarse $\mathrm{y}$ rotarse usando las transformaciones de ángulos de Euler [1][2].

$$
\dot{\eta}=J(\eta) \nu
$$

donde $\boldsymbol{J}(\eta)$ es la matriz de transformación de ángulos de Euler.

Las ecuaciones dinámicas se obtienen aplicando las leyes de Newton que determinan el movimiento del vehículo en el_mar [11]. Para ello es necesario asumir que el vehículo es_un cuerpo rígido y el sistema de referencia fijado en tierra es_inercial [13].

Agrupando las expresiones que caracterizan la dinámica de_un cuerpo rígido que se mueve en un medio líquido se obtiene la siguiente expresión:

$$
M_{C} \dot{\nu}+C_{C}(\nu) \nu=\tau
$$

donde $\boldsymbol{M}_{\boldsymbol{C}}$ representa a la matriz de masa del cuerpo rígido [2] y $C_{C}$ constituye la matriz que agrupa los términos de fuerzas centrípetas y de Coriolis.

El término $\tau$ designa a las fuerzas y momentos externos a causa de la interacción con el fluido y elementos medioambientales como el viento, las corrientes y las olas, así como la acción provocada por los actuadores. La fuerza generalizada aplicada a la embarcación está dada por [2]:

$$
\tau=\tau_{\boldsymbol{H}}-g(\eta)+\tau_{\boldsymbol{W}}
$$

donde:

- $\tau_{H}$ constituye la fuerza generalizada resultante de la interacción con un fluido.

- $g(\eta)$ representa la fuerza resultante del campo gravitacional terrestre y las presiones hidrostáticas. Es relativamente fácil de calcular y depende de una forma estática de la posición $\eta$.

- $\tau_{W}$ es la fuerza resultante de la interacción con el viento. Se calcula a partir de la posición y la velocidad del catamarán.

Las fuerzas hidrodinámicas generalizadas $\tau H$ aplicadas a la embarcación están dadas por:

$$
\tau_{\boldsymbol{H}}=\tau_{\boldsymbol{H} \boldsymbol{N} \boldsymbol{W}}\left(\nu_{r}, \dot{\nu}_{r}, n_{c}, n_{d}, \delta r\right)+\tau_{\boldsymbol{F} \boldsymbol{K}}\left(\chi_{\boldsymbol{F}}\right)
$$

donde:

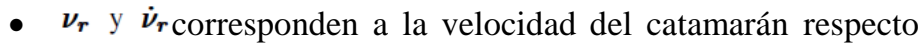
al fluido irrotacional y su derivada en el tiempo.

- $\boldsymbol{\tau}_{H N W}$ representa las fuerzas hidrodinámicas generalizadas aplicadas al catamarán en ausencia de olas.

$$
\tau_{H N W}=\left[\tau_{1_{H N W}}, \tau_{2_{H N W}}\right]^{T}=\tau_{A}+\tau_{D}
$$

donde $\tau_{A}$ representa las fuerzas por masas añadidas y $\tau_{D}$ la acción de los términos lineales y cuadráticos de las fuerzas amortiguadoras.

- $\tau_{F K}$ representa la fuerza generalizada de Froude-Krylov, generado por el campo de presiones de las olas.

$$
\tau_{F K}=M_{F} \chi_{F}
$$

siendo $\tau_{F}$ la aceleración del fluido medida en $\{U\}$ y expresada en $\{C\}$ y $M F$ la matriz de masa del fluido que ocuparía el volumen del cuerpo según 8 :

$$
\boldsymbol{M}_{\boldsymbol{F}}=\left[\begin{array}{cccccc}
m_{F} & 0 & 0 & 0 & 0 & 0 \\
0 & m_{F} & 0 & 0 & 0 & 0 \\
0 & 0 & m_{F} & 0 & 0 & 0 \\
0 & -m_{F} z_{B} & m_{F} y_{B} & 0 & 0 & 0 \\
m_{F} z_{B} & 0 & -m_{F} x_{B} & 0 & 0 & 0 \\
-m_{F} y_{B} & m_{F} x_{B} & 0 & 0 & 0 & 0
\end{array}\right]
$$

donde $x_{B}, y_{B}$ y $z_{B}$ son las respectivas posiciones del centro de flotabilidad $r_{B}=\left[x_{B}, y_{B}, z_{B}\right]^{T}$ respecto al origen de coordenadas $O_{C}$.

En la Figura 2, se presenta el diagrama general que representa la dinámica del catamarán, el cual está formado por cinco bloques principales.

\section{CALCULO DE LOS PARÁMETROS DEL MODELO}

\section{IV.1 PARÁMETROS DEL CUERPO RÍGIDO}

Atendiendo a la disposición estructural del catamarán de (SIMPRO), y a las consideraciones realizadas, la matriz $M_{C}$ puede ser calculada como: 


$$
M_{\boldsymbol{C}}=\left[\begin{array}{cccccc}
m & 0 & 0 & 0 & 0 & 0 \\
0 & m & 0 & 0 & 0 & 0 \\
0 & 0 & m & 0 & 0 & 0 \\
0 & 0 & 0 & I_{x} & 0 & -I_{x z} \\
0 & 0 & 0 & 0 & I_{y} & 0 \\
0 & 0 & 0 & -I_{x z} & 0 & -I_{z}
\end{array}\right]
$$

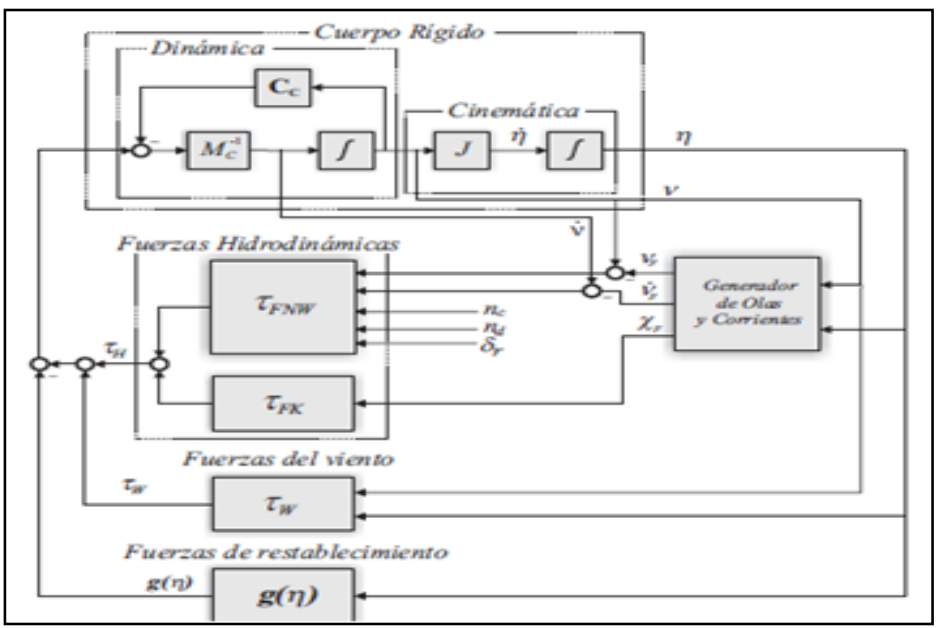

Figura 2: Diagrama general para el modelo dinámico del catamarán. Fuente: Los Autores, (2015).

y la matriz $C_{C}$ puede ser obtenida como:

$\left.C_{\boldsymbol{C}}=\left[\begin{array}{cccccc}0 & -m r & m q & 0 & 0 & 0 \\ m r & 0 & -m p & 0 & 0 & 0 \\ -m q & m p & 0 & 0 & 0 & 0 \\ 0 & 0 & 0 & 0 & -I_{x z}+I_{z} r & -I_{y} q \\ 0 & 0 & 0 & I_{x z} p-I_{z} r & 0 & -I_{x z} r+I_{x} p \\ 0 & 0 & 0 & I_{y} q & I_{x z} r-I_{x} p & 0\end{array}\right]\right)$

\section{IV.2 TÉRMINOS HIDRODINÁMICOS}

Para calcular las fuerzas y momentos totales de origen hidrodinámico aplicados al catamarán $\tau_{H N W}$, es necesario analizar por separado las fuerzas y momentos en cada uno de los elementos estructurales que forman parte de la embarcación e interactúan con el mar. En el caso del catamarán de SIMPRO, los cascos y los motores constituyen los elementos a analizar.

1) Cascos: Para los cascos, las fuerzas de masas añadidas y amortiguamiento, así como resistencia al fluido juegan un papel decisivo en el comportamiento dinámico del vehículo.

Los coeficientes de masas añadidas se pueden obtener aproximando cada flotador a un semi elipsoide con dimensiones aumentadas en un $20 \%$, cuyos semiejes se calculan a partir de las características físicas de la embarcación.

La fuerza generalizada de las masas añadidas se encuentra dada por las ecuaciones de energía cinética planteadas por Krichhoff [14]. Esta fuerza se define como:

$$
\tau_{A}=-M_{A} \dot{\nu}-C_{A^{\nu}}
$$

donde $M A$ representa la matriz de masas añadidas del cuerpo rígido:

$$
M_{\boldsymbol{A}}=\operatorname{diag}\left\{A_{11}^{\text {elip }}, A_{22}^{\text {elip }}, A_{33}^{\text {elip }}, A_{44}^{\text {elip }}, A_{55}^{\text {elip }}, A_{66}^{\text {elip }}\right\}
$$

y la matriz $C_{A}$ queda de la forma:

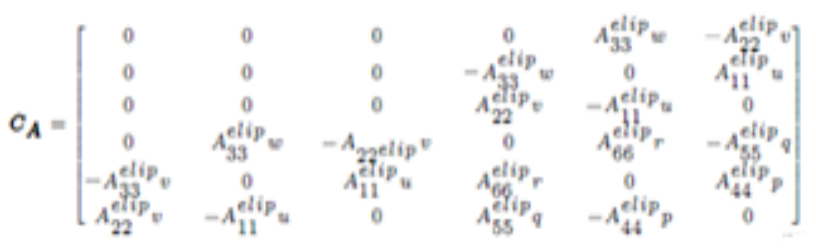

El cálculo de cada uno de los coeficientes de los términos anteriores pueden ser encontrados en [14].

De acuerdo a los planteamientos analíticos para la obtención de los términos de amortiguamiento expresados en [10] y de resistencia abordados en [2], se pueden obtener las expresiones de los cascos dadas por los coeficientes dimensionales $X_{u}^{c}, X_{v r}^{c}, \ldots, N_{r|r|}^{c}$.

$$
\begin{aligned}
& X_{u}^{c}=-A_{11}^{c} \\
& X_{v r}^{c}=A_{22}^{c} \\
& X_{w q}^{c}=-A_{33}^{c} \\
& Y_{v}^{c}=-A_{22}^{c} \\
& Y_{w q}^{c}=A_{33}^{c} \\
& Y_{u v}^{c}=\frac{1}{2} \rho L T Y_{v}^{\prime} c \\
& Y_{u r}^{c}=-A_{11}^{c}+\frac{1}{2} \rho L^{2} T Y_{r}^{\prime} c
\end{aligned}
$$$$
Z_{v p}=-A_{22}^{c}
$$$$
Z_{u q}=A_{11}^{c}
$$$$
\begin{aligned}
& K_{\dot{p}}^{c}=-A_{44}^{c} \\
& K_{v w}^{c}=A_{22}^{c}-A_{33}^{c}
\end{aligned}
$$$$
M_{\dot{q}}^{c}=-A_{55}^{c}
$$$$
M_{u w}^{c}=A_{33}^{c}-A_{11}^{c}
$$$$
N_{\dot{r}}^{c}=-A_{66}^{c}
$$$$
N_{p q}^{c}=A_{44}^{c}-A_{55}^{c}
$$$$
N_{u v}^{c}=A_{11}^{c}-A_{22}^{c}+\frac{1}{2} \rho L^{2} T N_{v}^{\prime c}
$$

$$
\begin{aligned}
& X_{u}^{c}=-R_{u}^{c} \\
& X_{u}^{c}=-R_{u}^{c} \\
& X_{v v}^{c}=\frac{1}{2} \rho L^{2} X_{v v}^{\prime} \\
& Y_{v|v|}^{c}=\frac{1}{2} \rho L T Y_{v \mid}^{\prime} c \\
& Y_{v|r|}^{c}=\frac{1}{2} \rho L T^{2} Y_{v|r|}^{\prime} c \\
& Y_{r|r|}^{c}=\frac{1}{2} \rho L T^{3} Y_{r|r|}^{\prime} c \\
& Z_{\dot{w}}^{c}=-A_{33}^{c} \\
& K_{q r}^{c}=A_{55}^{c}-A_{66}^{c} \\
& M_{p r}^{c}=A_{66}^{c}-A_{44}^{c} \\
& N_{u r}^{c}=\frac{1}{2} \rho L^{3} T N_{r}^{\prime c} \\
& N_{v^{2} r / u}^{c}=\frac{1}{2} \rho L^{3} T N_{v v r}^{\prime} c \\
& N_{r|r|}^{c}=\frac{1}{2} \rho L T^{4} N_{r|r|}^{\prime}
\end{aligned}
$$

2) Motores: Las fuerzas y momentos originados por las hélices se calculan en base a los coeficientes de impulso $K_{T}$ y binario $K_{Q}$, así como de los parámetros estructurales fundamentales de las hélices [2].

$$
\begin{aligned}
X_{h} & =X_{u u}^{h} u_{h}^{2}+X_{u n}^{h} u_{h} n+X_{n n}^{h} n^{2} \\
Y_{h} & =Y_{u v}^{h} u_{h} v_{h}+Y_{v n}^{h} v_{h} n \\
N_{h} & =N_{u r}^{h} u_{h} r_{h}+N_{r n}^{h} r_{h} n
\end{aligned}
$$

Los términos $X_{u u}^{h} \ldots N_{n n}^{h}$ pueden ser hallados utilizando las expresiones que aparecen en [2].

Los motores definen las fuerzas y momentos de control.

Todo motor fuera de borda típico, incluye una pequeña superficie vertical, que genera una fuerza lateral que provee estabilidad y control [15].

Las fuerzas y momentos que se producen debido al ángulo de deflexión de los motores pueden ser expresados mediante el producto vectorial del vector de fuerzas que se encuentran en función del ángulo de deflexión y la distancia a la que se encuentra el actuador respecto al origen $\mathrm{O}_{C}$ [16]. De acuerdo con la observado en la literatura [2][15], se obtienen las expresiones que definen la acción debido al ángulo de deflexión de los motores: 


$$
\begin{aligned}
X_{\delta r} & =\left(X_{u u}^{\delta r} u_{\delta r}^{2}+X_{u n}^{\delta r} u_{\delta r} n+X_{n n}^{\delta r} n^{2}\right) \cos (\delta r) \\
Y_{\delta r} & =\left(Y_{u n}^{\delta r} u_{\delta r} n+Y_{n n}^{\delta r} n^{2}\right) \sin (\delta r) \\
Z_{\delta r} & =K_{\delta r}=M_{\delta r}=0 \\
N_{\delta r} & =\left(N_{u n}^{\delta r} u_{\delta r} n+N_{n n}^{\delta r} n^{2}\right) \sin (\delta r) x_{h}- \\
& =\left(N_{u u}^{\delta r} u_{\delta r}^{2}+N_{u n}^{\delta r} u_{\delta r} n+N_{n n}^{\delta r} n^{2}\right) \cos (\delta r) y_{h}
\end{aligned}
$$

Los términos $X_{u u}^{\delta r} \ldots N_{n n}^{\delta r}$ pueden ser calculados a partir de las expresiones que aparecen en [2].

\section{IV.3 FUERZAS Y MOMENTOS HIDRODINÁMICOS TOTALES APLICADOS AL CATAMARÁN}

Para estimar las fuerzas y momentos aplicados al catamarán al moverse en un fluido, es necesario expresar cada una de las contribuciones a partir de una referencia en particular en función de las velocidades de traslación y rotación propias. No obstante, estas fuerzas y momentos deben expresarse según el sistema de referencia del catamarán. Para ello, es necesario establecer valores de desplazamiento en los distintos ejes.

Las coordenadas de origen de las referencias de los elementos particulares en relación a la referencia general representadas como $x_{c 1}, y_{c 1}, \ldots, x_{h 2}, y_{h 2}$ se encuentra en la Tabla 5.

Tabla 5: Coordenadas de origen de cada elemento con respecto a la referencia del catamarán.

\begin{tabular}{ccccc}
\hline & Casco 1 & Casco 2 & Hélice y timón 1 & Hélice y timón 2 \\
\hline $\mathrm{x}$ & 0 & 0 & $x_{h 1}$ & $x_{h 2}$ \\
\hline $\mathrm{y}$ & $y_{c 1}$ & $y_{c 2}$ & $y_{h 1}$ & $y_{h 2}$ \\
\hline $\mathrm{z}$ & 0 & 0 & 0 & 0 \\
\hline
\end{tabular}

Fuente: Los Autores, (2015).

Las velocidades de rotación de las hélices $n_{1}$ y $n_{2}$ se sustituyen por sus velocidades respectivas según sus modos de trabajo:

$$
n_{c}=\frac{n_{1}+n_{2}}{2} \quad n_{d}=\frac{n_{1}-n_{2}}{2}
$$

Los coeficientes dimensionales de las ecuaciones de las fuerzas y momentos hidrodinámicos son:

$$
\begin{aligned}
& X_{\dot{u}}=2 X_{\dot{u}}^{c} \\
& X_{v r}=2 X_{v r}^{c} \\
& X_{v^{2}}=2 X_{v v}^{c} \\
& X_{u n_{c}}=2 X_{u n}^{h} \\
& X_{w p}=2 X_{w p}^{c} \\
& X_{u^{2}}=2 X_{u u}^{h} \\
& X_{r^{2}}=2 X_{u u}^{h} y_{h}^{2} \\
& X_{u n_{d} \delta r}=2 X_{u n d}^{d r} \\
& X_{n_{d} n_{d} \delta r}=2 X_{n_{d} n_{d}}^{d r} \\
& Y_{\dot{v}}=2 Y_{\dot{v}} \\
& Y_{u r}=2 Y_{u r}^{c}+2 Y_{u v}^{h} x_{h} \\
& Y_{u v}=2 Y_{u v}^{c}+2 Y_{u v}^{h} \\
& Y_{w q}=2 Y_{w q}^{c} \\
& Y_{v|r|}=2 Y_{v|r|}^{c} \\
& Y_{u n_{c} \delta r}=2 Y_{u n c}^{d r} \\
& Y_{n_{d} n_{d} \delta r}=2 Y_{n_{d} n_{d}}^{d r} \\
& \\
& Z_{v p}=2 Z_{v p}^{c} \\
& Z_{\dot{w}}=2 Z_{\dot{w}}^{c}
\end{aligned}
$$

\begin{tabular}{|c|c|}
\hline$K_{\dot{p}} \dot{p}=2 K_{\dot{p}}^{c}$ & $K_{v w}=2 K_{v w}^{c}$ \\
\hline$K_{u v}=\rho T L^{3} K_{v}^{\prime c o}$ & $K_{v v}=\rho T L^{3} K_{v v}^{\prime c o}$ \\
\hline$K_{v|r|}=\rho T L^{4} K_{v|r|}^{\prime c o}$ & $K_{\frac{v r^{2}}{u}}=\rho T L^{5} K_{v r r}^{\prime} c o$ \\
\hline$K_{\frac{r^{3}}{u}}=\rho T L^{6} K_{r r r}^{\prime c o}$ & $K_{\frac{v^{2} r}{u}}^{u}=\rho T L^{4} K_{r v v}^{\prime c o}$ \\
\hline$K_{u p}^{u}=\rho T L^{4} K_{p}^{\prime c o}$. & $K_{p|p|}^{u}=\rho T L^{5} K_{p|p|}^{\prime c o}$ \\
\hline$K_{p u|p u|}=\rho T L^{5} K_{p u|p u|}^{\prime c o}$ & $K_{\dot{r}}=2 X_{\dot{u}}^{c} y_{c}^{2}$ \\
\hline$K_{p|p|}=\rho T L^{5} K_{p|p|}^{\prime c o}$ & $K_{r|r|}=\rho T L^{5} K_{r|r|}^{\prime c o}$ \\
\hline$K_{v|v|}=\rho T L^{3} K_{v|v|}^{\prime \prime c o}$ & $K_{r|v|}=\rho T L^{4} K_{r|v|}^{\prime c o}$ \\
\hline$K_{r|r|}=\rho T L^{5} K_{r|r|}^{\prime c o}$ & $K_{p^{3}}=\rho T L^{6} K_{p p p}^{\prime c o}$ \\
\hline$K_{p r}=2 K_{p r}^{c}$ & \\
\hline $\begin{array}{l}M_{q}=2 M_{\phi}^{c} \\
M_{p r}=2 M_{p r}^{c}\end{array}$ & $M_{\dot{r}}=2 X_{\dot{u}}^{c} y_{c}^{2}$ \\
\hline$N_{\dot{r}}=2 N_{\dot{r}}^{c}+2 X_{\dot{u}}^{c} y_{c}^{2}$ & $N_{v^{2} r / u}=2 N_{v^{2} r / u}^{c}$ \\
\hline$N_{p q}=2 N_{p q}^{c}$ & $N_{\mathrm{u}^{2} \mathrm{r}}=6 X_{\mathrm{u}^{3}}^{c} y_{c}^{2}$ \\
\hline$N_{u v}=2 N_{u v}^{c}+2 Y_{u v}^{h} x_{h}$ & $N_{r|r|}=2 N_{r|r|}^{c}$ \\
\hline$N_{r}=2 X_{u}^{c} y_{c}^{2}$ & $N_{n_{c} n_{c} \delta r}=2 N$ \\
\hline$N_{v n_{c}}=2 Y_{v n}^{h} x_{h}$ & $N_{n_{c} n_{d}}=-4 X_{n_{n}}^{h} y_{h}$ \\
\hline$N_{u n_{c} \delta r}=2 N_{u n c}^{d r} x_{h}$ & $N_{u n_{d} \delta r}=2 N_{u n d}^{d r} x_{h}$ \\
\hline$N_{r n_{c}}=2 N_{r n}^{h}+2 X_{u n}^{h} y_{h}^{2}+2 Y_{v n}^{h} x_{h}^{2}$ & $N_{u n_{d}}=-2 X_{u n}^{h} y_{h}$ \\
\hline$N_{n_{d} n_{d} \delta r}=2 N_{n_{d} n_{d}}^{d r} x_{h}$ & $N_{v r^{2} / u}=2 N_{v r}^{c}$ \\
\hline $\begin{array}{l}N_{u r}=2 N_{u r}^{c}+2 N_{u r}^{h}+2 Y_{u v}^{h} x_{h}^{2}+ \\
4 X_{u u}^{h} y_{h}^{2}\end{array}$ & $N_{r^{3}}=2 X_{u^{3}}^{c} ?$ \\
\hline
\end{tabular}$$
X_{u}=2 X_{u}^{c}
$$$$
X_{u^{3}}=2 X_{u^{3}}^{c}
$$$$
X_{u r^{2}}=6 X_{u^{3}} y_{c}^{2}
$$$$
X_{n_{c} n_{c}}=2 X_{n n}^{h}
$$$$
X_{n_{c} n_{c} \delta r}=2 X_{n_{c} n_{c}}^{d r}
$$$$
X_{u n_{c} \delta r}=2 X_{u n c}^{d r}
$$$$
X_{r n_{d}}=-2 X_{u n}^{h} y_{h}
$$$$
X_{u^{2} \delta r}=2 X_{u u}^{d r}
$$$$
X_{n_{d} n_{d}}=2 X_{n n}^{h}
$$$$
Y_{v n_{c}}=2 Y_{v n}^{h}
$$$$
Y_{v|v|}=2 Y_{v|v|}^{c}
$$$$
Y_{r|r|}=2 Y_{r|r|}^{c}
$$$$
Y_{r n_{c}}=2 Y_{v n}^{h} x_{h}
$$$$
Y_{n_{c} n_{c} \delta r}=2 Y_{n_{c} n_{c}}^{d r}
$$$$
Y_{u_{\text {d }} \delta r}=2 Y_{u n d}^{d r}
$$$$
Z_{u q}=2 Z_{u q}^{c}
$$$$
Z_{q}=2 \rho L^{2} T Z_{q}^{\prime} m
$$

\section{IV.4 TÉRMINOS GRAVITACIONALES E HIDROSTÁTICOS}

En la hidrodinámica, las fuerzas gravitacionales y de flotabilidad, o hidrostáticas, se conocen como fuerzas restauradoras [14]. Las fuerzas restauradoras son asociadas usualmente con la estabilidad metacéntrica. Se considera que los cambios en el fluido desplazado debido a las variaciones que se producen en $z$ son pequeños y que $\phi, \theta$ y $z$ son pequeños. Atendiendo a estas consideraciones se pueden definir las fuerzas gravitacionales y de flotabilidad como:

$$
\boldsymbol{g}(\boldsymbol{\eta})=\left[\begin{array}{c}
\mathbf{0}_{\mathbf{3 x \mathbf { 1 }}} \\
B_{f} \overline{G M}_{T} \phi \\
B_{f} \overline{G M}_{L} \theta \\
0
\end{array}\right]
$$

donde:

- $B_{f}$ representa la fuerza de flotabilidad definida por:

$$
B_{f}=\rho g \nabla
$$

siendo $\nabla$ el volumen del vehículo sumergido y $\rho$ es la densidad del agua.

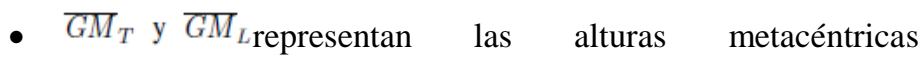
transversal y longitudinal, las cuales definen la distancia entre el metacentro y el centro de gravedad $r_{G}$.

\section{5 FUERZAS Y MOMENTOS GENERADOS POR EL VIENTO}

Las fuerzas y momentos provocados por el viento y que afectan la dinámica del catamarán se modelan a partir de la siguiente ecuación: 


$$
\boldsymbol{\tau}_{W}=\left[\begin{array}{c}
X_{W} \\
Y_{W} \\
\mathbf{0}_{\mathbf{3} \mathbf{1}} \\
N_{W}
\end{array}\right]=\left[\begin{array}{c}
\frac{1}{2} \rho_{w} V_{R}^{2} A_{T} C_{X}\left(\gamma_{R}\right) \\
\frac{1}{2} \rho_{w} V_{R}^{2} A_{L} C_{Y}\left(\gamma_{R}\right) \\
\mathbf{0}_{\mathbf{3 x 1}} \\
\frac{1}{2} \rho_{w} V_{R}^{2} L C_{N}\left(\gamma_{R}\right)
\end{array}\right]
$$

donde:

- $\quad \rho_{w}$ representa la densidad del aire.

- $A_{T}, A_{L}$ y $L$ definen el área transversal y lateral, así como la longitud del vehículo.

- $V_{w} \mathrm{y} \phi_{w}$ representan la velocidad y la dirección del viento.

- $V_{R}$ y $\gamma_{R}$ representan el módulo y dirección de la velocidad del viento relativa al vehículo.

- $C_{X}, C_{Y}$ y $C \mathrm{~N}$ definen los coeficientes de torque y fuerzas aerodinámicas hallados a partir de datos de pruebas experimentales analizados mediante técnicas de regresión múltiple [11]. según:

Los términos del viento relativos al vehículo se calculan

$$
\begin{aligned}
& V_{R}=\sqrt{u_{r W}^{2}+v_{r W}^{2}} \\
& \gamma_{R}=-\operatorname{atan} 2\left(v_{r W}, u_{r W}\right)=\psi_{w}-\psi
\end{aligned}
$$

donde $u_{r W}$ y $v_{r W}$ son las componentes de la velocidad del viento relativa al vehículo, y son calculados por:

$$
\begin{aligned}
& u_{r W}=V_{W} \cos \gamma_{R}-u \\
& v_{r W}=V_{W} \sin \gamma_{R}-v
\end{aligned}
$$

\section{IV.6 GENERADOR DE OLAS Y CORRIENTES}

Las fuerzas y momentos hidrodinámicos aplicados al catamarán dependen de la velocidad relativa $\nu_{\boldsymbol{r}}$ y su respectiva derivada en el tiempo $\dot{\boldsymbol{\nu}}_{\boldsymbol{r}}$. Estos elementos dependen de las componentes $u_{F}$ y $v_{F}$ de la velocidad del fluido en ausencia del catamarán, así como de sus respectivas derivadas. Estos términos son obtenidos por:

$$
\begin{gathered}
\boldsymbol{\nu}_{\boldsymbol{F}}=\boldsymbol{J}^{-1}(\boldsymbol{\eta})\left[\begin{array}{c}
v_{x F} \\
v_{y F} \\
\mathbf{0}_{4 x 1}
\end{array}\right] \\
\dot{\boldsymbol{\nu}}_{\boldsymbol{F}}=\boldsymbol{J}^{-1}(\boldsymbol{\eta})\left[\begin{array}{c}
v_{x F} \\
v_{y F} \\
\mathbf{0}_{4 x 1}
\end{array}\right]+\boldsymbol{\chi}_{\boldsymbol{F}}+ \\
\boldsymbol{J}^{-1}(\boldsymbol{\eta}) \boldsymbol{P}\left[\begin{array}{ccc}
\frac{\partial v_{x}}{\partial x_{u}} & \frac{\partial v_{x}}{\partial y_{u}} & 0 \\
\frac{\partial v_{y}}{\partial x_{u}} & \frac{\partial v_{y}}{\partial y_{u}} & 0 \\
0 & 0 & 0
\end{array}\right] \boldsymbol{P}^{\boldsymbol{T}} \boldsymbol{J}(\boldsymbol{\eta}) \boldsymbol{\nu} \\
\boldsymbol{\chi} \boldsymbol{F}=\boldsymbol{J}^{-1}(\boldsymbol{\eta})\left[\begin{array}{c}
a_{x F} \\
a_{y F} \\
\mathbf{0}_{4 x 1}
\end{array}\right]
\end{gathered}
$$

donde:

- $v_{x F}$ y $v_{y F}$ son las componentes de velocidad del fluido en ausencia del catamarán medidas en $\{U\}$ y definidos por:

$$
\begin{aligned}
& v_{x F}=V_{F} \cos \psi_{c} \\
& v_{y F}=V_{F} \sin \psi_{c}
\end{aligned}
$$

siendo $V F$ la velocidad de las corrientes y $\psi c$ el ángulo de dirección de las mismas.

- $a_{x F}$ y $a_{y F}$ son las componentes de aceleración del fluido en ausencia del catamarán medidas en $\{U\}$.
- $\boldsymbol{P}$ es una matriz auxiliar utilizada en los cálculos matemáticos, definida como $P=\left[I_{3 \times 3}, 0_{3 \times 3}\right]^{T}$.

Las velocidades del fluido en ausencia del vehículo, son obtenidas a partir de la superposición de $N$ ondas planas monocromáticas con una corriente uniforme y estacionaria.

Las componentes horizontales de la velocidad y aceleración del fluido, así como sus derivadas parciales están dadas por las expresiones que aparecen en [2].

\section{VALORES NUMÉRICOS DEL MODELO DINÁMICO DEL \\ CATAMARÁN}

\section{V.1 TÉRMINOS DEL CUERPO RÍGIDO E HIDROSTÁTICOS}

Los datos presentados en la Tabla 2 sirven de base para el cálculo de los elementos del cuerpo rígido. La matrices de cuerpo rígido y de Coriolis son obtenidas según (8) y (9):

$$
\boldsymbol{M}_{\boldsymbol{C}}=\left[\begin{array}{cccccc}
4266 & 0 & 0 & 0 & 0 & 0 \\
0 & 4266 & 0 & 0 & 0 & 0 \\
0 & 0 & 4266 & 0 & 0 & 0 \\
0 & 0 & 0 & 2820 & 0 & -680 \\
0 & 0 & 0 & 0 & 1450 & 0 \\
0 & 0 & 0 & -680 & 0 & -1040
\end{array}\right]
$$

$$
O_{O}=\left[\begin{array}{cccccc}
0 & -4266 r & 4266 q & 0 & 0 & 0 \\
4266 r & 0 & -4266 p & 0 & 0 & 0 \\
-4266 q & 4266 p & 0 & 0 & 0 & 0 \\
0 & 0 & 0 & 0 & 1040 \mathrm{r}-680 \mathrm{p} & -1450 \mathrm{q} \\
0 & 0 & 0 & 680 \mathrm{p}-1040 \mathrm{r} & 0 & 2820 \mathrm{p}-640 \mathrm{r} \\
0 & 0 & 0 & 1450 \mathrm{q} & 640 \mathrm{r}-2820 \mathrm{p} & 0
\end{array}\right]
$$

El vector de fuerzas y momentos gravitacionales definido en (15) resulta:

$$
\boldsymbol{g}(\boldsymbol{\eta})=\left[\begin{array}{c}
\mathbf{0}_{\mathbf{3 x \mathbf { 1 }}} \\
777236.4 \phi \\
20661.7 \theta \\
0
\end{array}\right]
$$

\section{V.2 TÉRMINOS HIDRODINÁMICOS}

Los coeficientes de masas añadidas del cuerpo rígido son: $X_{\dot{u}}=-173.4 \mathrm{~kg}, Y_{\dot{v}}=Z_{\dot{w}}=-4291.2 \mathrm{~kg}, K_{\dot{p}}=0 \mathrm{y} M_{\dot{q}}=N_{\dot{r}}=-26834 \mathrm{kgm}^{2} . \mathrm{A}$ partir de estos valores, las matrices de masas añadidas del cuerpo rígido y Coriolis quedan definidas, según (11) y (12):

$$
\boldsymbol{M}_{\boldsymbol{A}}=\left[\begin{array}{cccccc}
173.4 & 0 & 0 & 0 & 0 & 0 \\
0 & 4291.2 & 0 & 0 & 0 & 0 \\
0 & 0 & 4291.2 & 0 & 0 & 0 \\
0 & 0 & 0 & 0 & 0 & 0 \\
0 & 0 & 0 & 0 & 26834 & 0 \\
0 & 0 & 0 & 0 & 0 & 26834
\end{array}\right]
$$

$$
\boldsymbol{C}_{\boldsymbol{A}}=\left[\begin{array}{cccccc}
0 & 0 & 0 & 0 & 4291.2 w & -4291.2 v \\
0 & 0 & 0 & -4291.2 w & 0 & 173.4 u \\
0 & 0 & 0 & 4291.2 v & -173.4 u & 0 \\
0 & 4291.2 w & -4291.2 v & 0 & 26834 r & -26834 q \\
-4291.2 v & 0 & 173.4 u & 26834 r & 0 & 0 \\
4291.2 v & -173.4 u & 0 & 26834 q & 0 & 0
\end{array}\right]
$$

Un esfuerzo importante en el cálculo de los parámetros de las fuerzas y momentos actuantes sobre el catamarán, radica en la búsqueda de los coeficientes hidrodinámicos adimensionales. Para el caso del catamarán de SIMPRO han 
tenido que ser calculados por la vía analítica, utilizando las expresiones propuestas por Inoue en [10].

Los valores numéricos de los coeficientes dimensionales, referidos a las fuerzas y momentos hidrodinámicos correspondientes al catamarán de SIMPRO, se presentan a continuación:

$$
\begin{aligned}
& X_{\dot{u}}=-346.7756 \mathrm{~kg} \\
& X_{v r}=-8442.4 \mathrm{~kg}^{-2} \\
& X_{v^{2}}=-921.348 \mathrm{kgm}^{-2} \\
& X_{u n_{c}}=-5.4427 \mathrm{~kg} \\
& X_{r n_{d}}=5.1162 \mathrm{kgm}
\end{aligned}
$$$$
X_{n_{c} n_{c}}=0.7465 \mathrm{kgm}
$$$$
X_{n_{c} n_{c} \delta r}=0.7465 \mathrm{kgm}
$$$$
X_{u n_{c} \delta r}=-5.4427 \mathrm{~kg}
$$$$
X_{u}=-0.48 \mathrm{kgm}^{-1}
$$$$
X_{u^{3}}=-7.6 \mathrm{kgm}^{-2}
$$

Es válido aclarar que los términos cuyo valor numérico no aparece es porque son cero.

En el caso de la matriz de masas de fluido de FroudeKrylov, se calcula según (7), obteniéndose:

$$
\boldsymbol{M}_{\boldsymbol{F}}=\left[\begin{array}{cccccc}
4266 & 0 & 0 & 0 & 0 & 0 \\
0 & 4266 & 0 & 0 & 0 & 0 \\
0 & 0 & 4266 & 0 & 0 & 0 \\
0 & -1493.1 & 0 & 0 & 0 & 0 \\
1493.1 & 0 & 0 & 0 & 0 & 0 \\
0 & 0 & 0 & 0 & 0 & 0
\end{array}\right]
$$

\section{COMPORTAMIENTO DEL MODELO OBTENIDO}

En la realidad, los vehículos marinos están sometidos a efectos medioambientales como la acción de las corrientes, las olas y el viento. Estos elementos originan fuerzas y momentos que son determinantes en el comportamiento de los vehículos y en la forma en que siguen las trayectorias. Las olas y las corrientes influyen en las fuerzas y momentos hidrodinámicos, mientras que el viento afecta los movimientos de avance, desplazamiento lateral y guiñada.

En la Figura 3 se presenta en color negro la trayectoria en línea recta obtenida con el modelo sin considerar el efecto de las perturbaciones, mientras que en color gris se observa la salida del modelo teniendo en cuenta la acción de las olas, las corrientes marinas y el viento. Tal como se puede apreciar, las corrientes provocan una desviación en la trayectoria que debe seguir el vehículo. Durante la simulación la velocidad de giros de los motores se fijó en $60 \mathrm{rps}$, manteniendo a $\delta \mathrm{r}=0^{\circ}$. Las corrientes se simularon con valor de velocidad $\mathrm{VF}=0.5 \mathrm{~m} / \mathrm{s}$ y un ángulo de $\psi \mathrm{c}=30^{\circ}$. Por su parte, las afectaciones provocadas por el viento se simulan considerando una velocidad $\mathrm{VW}=0.5 \mathrm{~m} / \mathrm{s}$ y un ángulo de $\psi_{\mathrm{w}}=0^{\circ}$.

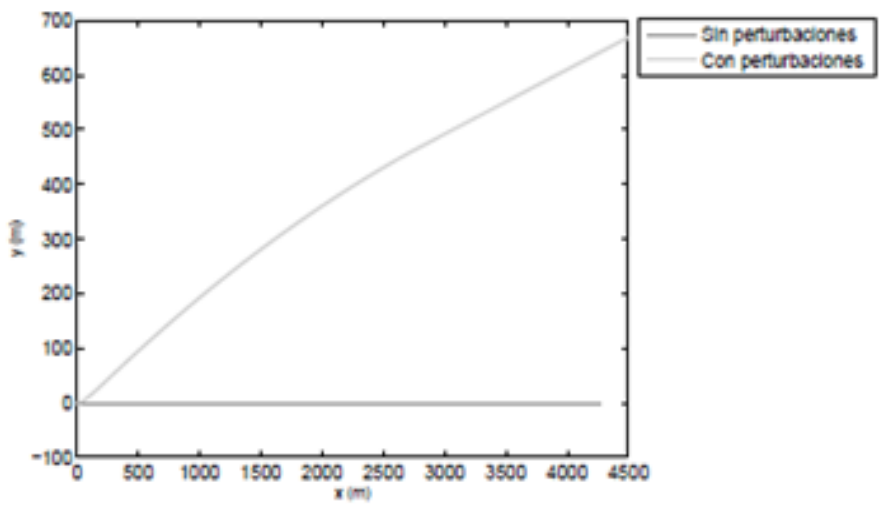

Figura 3: Simulación de una maniobra en línea recta con el modelo no lineal de $6 G D L$ con y sin perturbaciones.

Fuente: Los Autores, (2015).
En una maniobra circular, la forma de la trayectoria depende del ángulo de incidencia y de la velocidad con que las perturbaciones afecten al vehículo. En la Figura 4, en color negro se muestra la simulación del modelo sin perturbaciones y en color gris la simulación del modelo considerando el efecto de las perturbaciones marinas. Durante la simulación, los motores giraron a $60 \mathrm{rps}$ y tenían un ángulo de deflexión de $\delta r=30^{\circ}$. La velocidad de las corrientes utilizada en la simulación es de $V F=$ $0.2 \mathrm{~m} / \mathrm{s}$, mientras que el ángulo es de $\phi c=0^{\circ}$. El viento se simuló utilizando valores de $V W=0.5 \mathrm{~m} / \mathrm{s}$ y $\psi_{w}=0^{\circ}$.

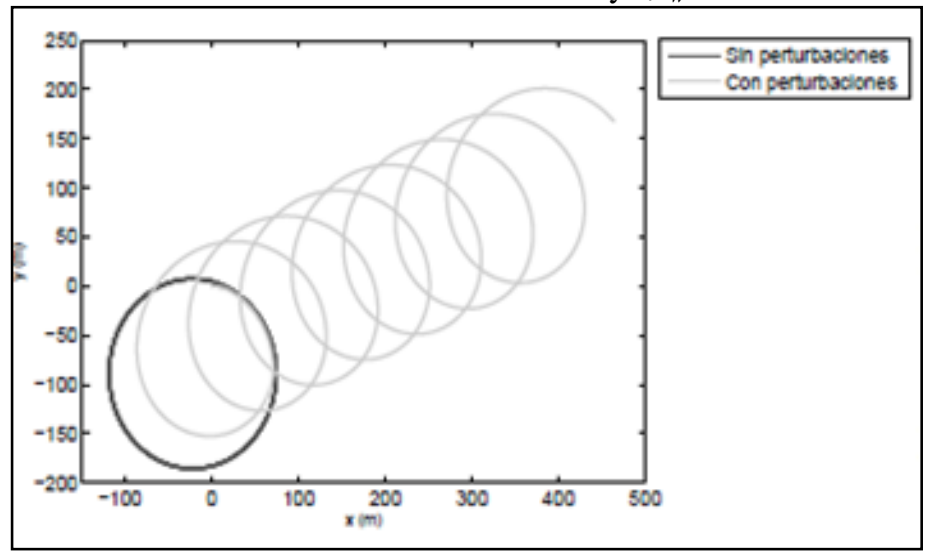

Figura 4: Simulación de una maniobra circular con el modelo no lineal de $6 G D L$ con y sin perturbaciones.

Fuente: Los Autores, (2015).

Las maniobras circulares también se producen se utilizan velocidades de giro diferentes en cada motor, de esta manera se produce una descompensación de fuerzas y momentos que provoca el cambio en la dirección del vehículo. En las Figuras 5 y 6 , se puede observar las componentes de posición y velocidad durante la simulación una maniobra circular provocada por las velocidades con que rotan los motores. Para provocar los giros en la trayectoria se coloca a un motor a trabajar a 60 rps y el otro a $10 \mathrm{rps}$, mientras que $\delta r=0^{\circ}$. La velocidad de las corrientes se mantuvo en $0.2 \mathrm{~m} / \mathrm{s}$ y la dirección de las mismas respecto al vehículo se estableció en $135^{\circ}$. Para simular los efectos provocados por el viento, se asigna un valor de $V W=0.1 \mathrm{~m} / \mathrm{s}$ y de $\psi c=10^{\circ}$
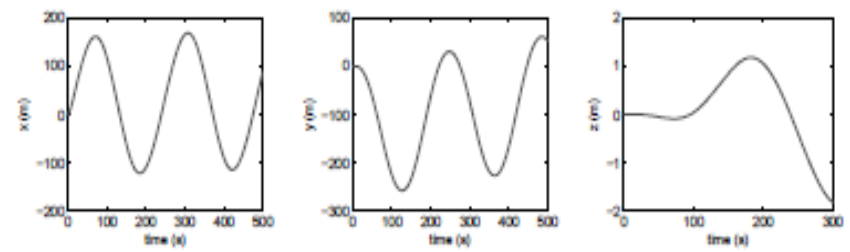

(a) Posición en el (b) Posición en el des- (c) Posición en la arfada. avance.

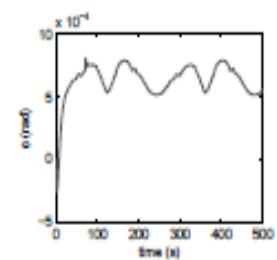
plazamiento lateral.
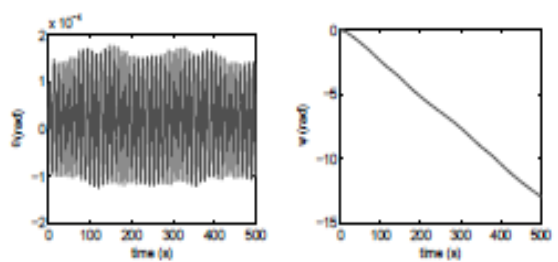

(d) Posición angular en (e) Posición angular en (f) Posición angular en el balanceo. el cabeceo. la guiñada.

Figura 5: Componentes de la posición del catamarán durante la simulación de una maniobra circular.

Fuente: Los Autores, (2015). 

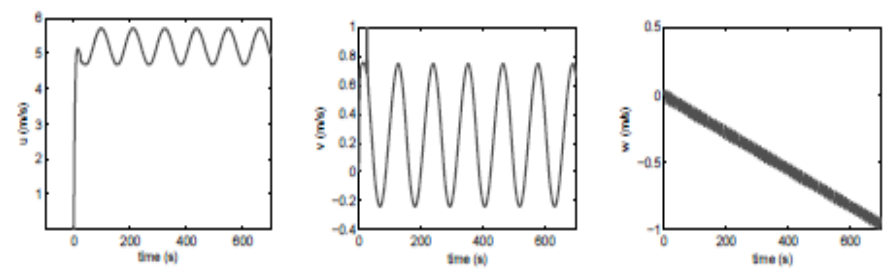

(a) Velocidad en el (b) Velocidad en el desavance.

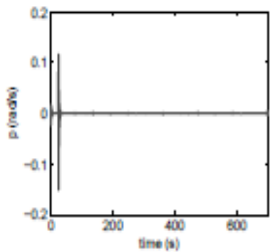
plazamiento latera.
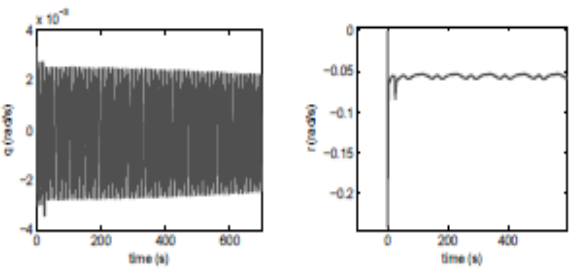

(d) Velocidad angular en el balanceo.

(e) Velocidad angular en el cabeceo.

Figura 6. Componentes de velocidad del catamarán durante la simulación de una maniobra circular.

Fuente: Los Autores, (2015).

Por último, se repite la simulación de la maniobra en zigzag, para visualizar las diferencia que provocan las perturbaciones marinas. La Figura 7, muestra la curva gris oscuro del modelo sin efectos perturbadores y la curva gris claro la trayectoria del modelo contaminada con la acción de los elementos medioambientales. El ángulo de deflexión de los motores obedece el comportamiento de una onda cuadrada cuya amplitud varía en el rango de -35 a 35 . Los datos medioambientales se mantienen en $V F=0.2 \mathrm{~m} / \mathrm{s}, \phi c=135^{\circ}, V F$ $=0.1 \mathrm{~m} / \mathrm{s}$ y $\phi w=10^{\circ}$. La inclusión en el modelo no lineal del catamarán de SIMPRO de los efectos que provocan el oleaje, el viento y las corrientes marinas permite recrear de manera más exactas las condiciones de operación del vehículo.

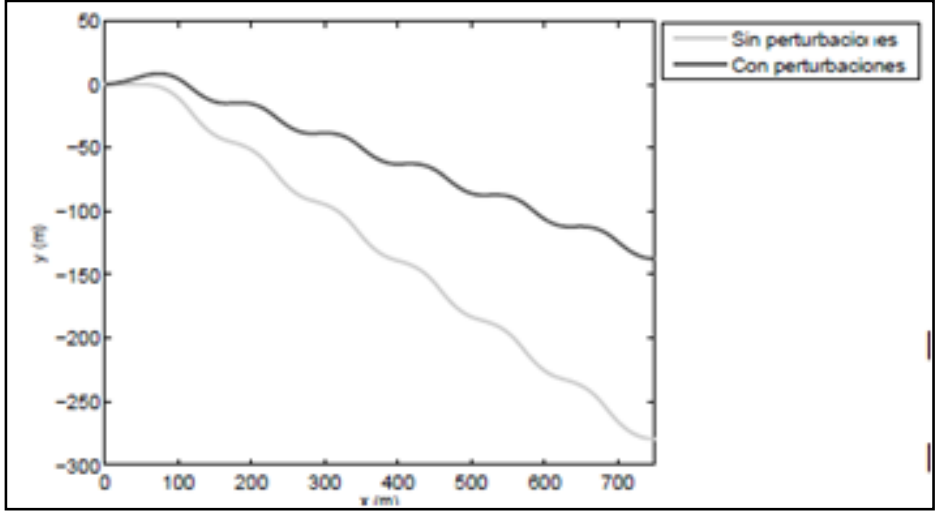

Figura 7: Modelo no lineal del Catamarán

Fuente: Los Autores, (2015).

En los resultados de las simulaciones se observa que para condiciones medioambientales no hostiles, el comportamiento del modelo no lineal de $6 G D L$ sigue siendo coherente con el desempeño que presentan vehículos de este tipo durante el desarrollo de maniobras en el mar.

\section{CONCLUSIONES}

En esta investigación, se determina un modelo no lineal de $6 G D L$ para el vehículo de superficie tipo catamarán de
SIMPRO, que representa adecuadamente las características dinámicas del vehículo. Con este resultado, es posible la implementación en un simulador de este tipo de vehículos.

El modelo se obtuvo mediante un procedimiento analítico y semi empírico, utilizando solamente algunos resultados de experimentos evidenciados en la literatura $y$ un costo computacional mínimo. El efecto de los actuadores, las olas y corrientes marinas, así como el viento, dotan al modelo dinámico del vehículo marino de un significado realismo a fin de cumplimentar con las exigencias de una aplicación de este tipo. La empresa SIMPRO se encuentra desarrollando el mundo virtual para acoplarla el modelo dinámico de 6 GDL del catamarán.

\section{REFERENCIAS}

[1] T. I. Fossen, Handbook of Marine Craft Hydrodynamics and Motion Control. New York, United States: John Wiley \& Sons., 2011.

[2] M. G. de Sousa, "Modelizaçã e controlo de um veículo oceanográfico autónomo," Master Thesis, Universidad Técnica de Lisboa. Instituto Superior Técnico, Lisboa, Portugal, July 2004.

[3] Y. Valeriano-Medina, A. Martínez, L. Hernández, H. Sahli, Y. Rodríguez, and J. R. Cañizares, "Dynamic model for an autonomous underwater vehicle based on experimental data," Mathematical and Computer Modelling of Dynamical Systems: Methods, Tools and Applications in Engineering and Related Sciences, vol. 19, no. 2, pp. 175-200, 2013.

[4] Y. Valeriano-Medina, "Modelado dinámico de un vehículo autónomo subacuático," Master Thesis, Universidad Marta Abreu de las Villas, Villa Clara, Cuba, January 2014.

[5] J. Fernández Ibarz, "Modelo de simulación y control dinámico para un catamarán submarino con seis grados de libertad," PHd Thesis, Universidad de la Coruña, Spain, April 2003.

[6] E. Lind and M. Meijer, "Simulation and control of submarines," Master Thesis, Department of Automatic Control, Lund University, Sweden, 2014.

[7] T. I. Fossen and A. Ross, Advances in unmanned marine vehicles. Great Britain: Peter Peregrinus LTD, 2006, vol. 69, ch. Nonlinear modelling, identification and control of UUVs, pp. $13-42$.

[8] J. E. da Silva, B. Terra, R. Martins, and J. B. de Sousa, "Modeling and simulation of the LAUV autonomous underwater vehicle," in 13th IEEE IFAC International Conference on Methods and Models in Automation and Robotics. Szczecin, Poland: IEEE Control Systems Society, 2007.

[9] D. Garcia-Garcia, Y. Valeriano-Medina, L. Hernández, and A. Martínez- Laguardia, "Wave filtering for heading control of an auv based on passive observer," Indian Journal of GeoMarine Sciences, vol. 41, no. 6, pp. 540-549, 2012. 
[10] S. Inoue, M. Hirano, and K. Kijima, "Hydrodynamic derivatives on ship manoeuvering," International Shipbuilding Progress, vol. 28, no. 321, pp. 112-125, May 1981.

[11] T. I. Fossen, Marine Control Systems Guidance, Navigation, and Control of Ships, Rigs and Underwater Vehicles. Norway: Marine Cybernetics, 2002.

[12] SNAME, "Nomenclature for treating the motion of a submerged body through a fluid," SNAME, New York, United States, Technical and Research Bulletin No. 1-5, April 1950.

[13] T. I. Fossen, "Nonlinear modelling and control of underwater vehicles,” PHd Thesis, NTNU, Norway, 1991.

[14] - Guidance and Control of Ocean Vehicles. New York, United States: John Wiley \& Sons., 1994.

[15] C. R. Sonnenburg, "Modeling, identification, and control of an unmanned surface vehicle," PHd Thesis, Universidad Estatal e Instituto Politécnico de Virginia, Virginia, United States, December 2012.

[16] T. I. Fossen, T. A. Johansen, and T. Pérez, Underwater vehicles. Vienna, Austria: InTech, 2008, ch. A survey of control allocation methods for underwater vehicles, pp. 109-128. 